\title{
Numerical simulation of plasma sheath expansion, with applications to plasma-source ion implantation
}

\author{
G. A. Emmert and M. A. Henry \\ Engineering Research Center for Plasma-Aided Manufacturing and Department of Nuclear Engineering \\ and Engineering Physics, University of Wisconsin-Madison, Madison, Wisconsin 53706
}

(Received 29 August 1991; accepted for publication 1 October 1991)

\begin{abstract}
In plasma-source ion implantation a target is pulse biased to a high negative voltage, forming an expanding plasma sheath. A numerical simulation model for the evolution of the sheath has been developed and compared successfully. with experimental results. The model is one dimensional (planar, cylindrical, or spherical). The time-dependent, self-consistent potential profile is calculated from Poisson's equation coupled with collisionless fluid equations for the ions and a Boltzmann assumption for the electrons. In addition to the density and potential profile, the simulation yields the ion current to the surface and the energy spectrum of the ions hitting the surface.
\end{abstract}

\section{INTRODUCTION}

Plasma-source ion implantation (PSII) is a new, nonline-of-sight technique for implanting ions into materials to modify their surface properties. ${ }^{1,2}$ In the PSII process, a target is immersed in a plasma and a negative voltage pulse is applied. A sheath between the plasma and the target forms and accelerates ions into the target. The ions are implanted in the target at energies up to the applied voltage. The implanted ions modify the surface properties for a wide range of materials. Information concerning the dose and energy spectrum of the implanted ions is needed to predict the modification to the surface. In addition, the current to the target determines target heating, which is important for temperature-sensitive materials.

Numerical simulation of expanding sheaths has been done by Widner et $a l^{3}$ using fluid equations for the ions coupled with the Boltzmann approximation for the electrons and Poisson's equation. They applied their simulation code to expanding sheaths formed by applying a potential suddenly to a metal surface; they found that the sheath initially grows faster than the ion acoustic speed, but then launches a density rarefaction wave when the sheath propagation velocity decreases to less than the ion acoustic speed. Chester, ${ }^{4}$ Lieberman, ${ }^{5}$ and Scheuer, Shamin, and Courad ${ }^{6}$ have treated expanding sheaths by assuming the sheath expands such that the ion current into the sheath is limited by the Child-Langmuir current. In this case, the current into the sheath is caused by the sheath motion uncovering ions and by any drift of the ions toward the sheath. Scheuer and co-workers pointed out that, for large values of the applied potential relative to the electron temperature, the sheath expands far into the plasma. The initial presheath is buried and the ions at the sheath edge are still stationary when the sheath comes by. This approximation is valid as long as the sheath propagation velocity is greater than the ion acoustic speed. When the propagation velocity drops below the ion acoustic speed, the density rarefaction wave sets up a plasma presheath which starts accelerating the ions toward the ion acoustic speed.

In PSII applications, the applied potential $\Phi$ is extremely large compared to the electron temperature $T_{e}$
( $e \Phi / k T_{e} \approx 10^{4}-10^{5}$ ) and the sheath expansion is rapid compared with the ion acoustic speed over the time span of the pulse. In addition, the finite rise time of the applied voltage pulse affects the sheath propagation and the energy of the ions incident on the target. Stewart and Lieberman ${ }^{7}$ recently analyzed the effect of the finite rise time by assuming that the flight time of an ion through the sheath is short compared with the rise time of the voltage pulse.

In this paper we present a numerical simulation model which, in its physical content, is identical to that used by Widner et al., but is implemented with a different numerical scheme; this allows the resulting computer code to run successfully with values of $e \Phi / k T_{e} \approx 10^{4}-10^{5}$ so that direct comparison with results from PSII experiments ${ }^{8}$ can be made. The ion motion is assumed to be collisionless and the ions are cold but are accelerated by the electric field. For the low pressures encountered in the PSII experiments of Shamim and co-workers, ${ }^{8}$ the collisionless ion approximation is reasonable. The time scale for the expansion of the sheath is long compared with the inverse of the electron plasma frequency. Consequently, electron inertia effects can be neglected and we assume that the electrons obey the Boltzmann approximation. This approximation allows the simulation code to jump over the electron time scale and solve the equations on the ion time scale without resorting to an artificial ion/electron mass ratio. The numerical procedure solves the ion fluid equations using a Lagrangian technique to determine the ion density. The electrostatic potential is obtained from Poisson's equation which has become nonlinear because of the Boltzmann approximation.

\section{FORMULATION OF THE SIMULATION MODEL}

The geometry considered is one dimensional with all variables functions of $r$, which is the radial direction in cylindrical or spherical coordinates, or $x$ in Cartesian coordinates. The simulation region extends from $r=a$ to $r$ $=b$. The target surfacc, where a given time-dependent potential is imposed, is at $a$. The surface at $b$ is taken to be in the plasma and far enough from $a$ such that the expanding sheath, which starts at $a$, does not reach $b$ during the 
duration of the simulation. The potential at $b$ is defined to be zero and remains so during the simulation.

The electric field is along the coordinate $r$; the ions are initially motionless and acquire motion only along $r$ upon acceleration by the electric field. We want the solution to the ion conservation equation

$$
\frac{\partial n}{\partial t}+\frac{\partial}{r^{p} \partial r}\left(r^{n} n v\right)=0,
$$

where $n$ is the ion density, $v$ is the ion fluid velocity, and the parameter $p$ equals 0,1 , or 2 for Cartesian, cylindrical, and spherical geometry, respectively. In order to solve Eq. (1) we introduce a set of marker particles, which mark the boundaries of fluid cells, equally spaced along $r$ between $a$ and $b$. The marker particles are initially stationary and move according to the equations of motion,

$$
\begin{aligned}
& \frac{d r}{d t}=v, \\
& m \frac{d v}{d t}=-e \frac{\partial \Phi}{\partial r},
\end{aligned}
$$

where $\Phi$ is the electrostatic potential, $m$ is the ion mass, and $-e$ is the electron charge. Given the solution to the equations of motion, the solution to the ion conservation equation is given by

$$
n_{k}(t) \Delta V_{k}\left(r_{k+1}, r_{k}, t\right)=n_{k}(0) \Delta V_{k}\left(r_{k+1}^{0}, r_{k}^{0}, 0\right),
$$

where $n_{k}(t)$ is the density in the cell bounded by marker particles $k$ and $k+1$ and $\Delta V_{k}$ is the volume of this cell. The volume depends on the position of the marker particles $k$ and $k+1$ and changes with time as the particles move. At $t=0$ these marker particles were at $r_{k}^{0}$ and $r_{k+1}^{0}$, respectively, the cell volume was $\Delta V_{k}\left(r_{k+1}^{0}, r_{k}^{0}, 0\right)$, and the density was $n_{k}(0)$.

The potential distribution is determined by Poisson's equation,

$$
\frac{\partial}{r^{p} \partial r}\left(r^{p} \frac{\partial \Phi}{\partial r}\right)=-\frac{e}{\epsilon_{0}}\left(n-n_{e}\right) .
$$

The electron density $n_{e}$ is given by the Boltzmann relation,

$$
n_{e}=n_{0} \exp \left(e \Phi / k T_{e}\right),
$$

where $n_{0}$ is the initial density in the plasma. Because the applied potential is large compared with the temperature, the electrons play no role in the sheath, but they are necessary to maintain quasineutrality in the plasma away from the sheath. Note that using the Boltzmann relation allows us to jump over the electron plasma frequency time scale and solve the equation on the ion time scale. There is a cost for this improvement; Poisson's equation has become nonlinear in the potential $\Phi$, which complicates the solution algorithm.

It is convenient to nondimensionalize Poisson's equation by introducing the variables $s=r /(b-a), n^{\prime}=n / n_{0}$, $\psi=-e \Phi / k T_{e}, L=b-a$. The Poisson's equation can be written

$$
\left(\frac{\lambda_{D}}{L}\right)^{2} \frac{\partial}{s^{2} \partial s}\left(s^{p} \frac{\partial \psi}{\partial s}\right)=-\left(n^{\prime}-e^{\psi}\right) .
$$

We solve Eq. (7) by iteration. We let $\phi$ be an initial guess for the solution and linearize Eq. (7) about this guess using

$$
e^{\psi}=e^{\phi} e^{\psi-\phi}=e^{\phi}(1+\psi-\phi),
$$

after neglecting higher-order terms. Then Eq. (7) becomes

$$
\left(\frac{\lambda_{D}}{L}\right)^{2} \frac{\partial}{s^{p} \partial s}\left(s^{p} \frac{\partial \psi}{\partial s}\right)-e^{\phi} \psi=-\left(n^{\prime}-e^{\phi}+\phi e^{\phi}\right) .
$$

This is now a linear Poisson's equation in the unknown $\psi$. We take $\phi$ from the $\psi$ of the previous time step, solve Eq. (8) to obtain a new $\psi$, set $\phi$ equal to this new $\psi$, and iterate until the process converges. Typically, it takes 2-3 iterations to converge. The solution of Eq. (8) is obtained by finite differencing it and using the Thomas algorithm ${ }^{9}$ to obtain $\psi$ from the specified boundary conditions. Thus we have both a fixed mesh and a set of marker particles defining moving cells of plasma; we typically use 100 marker particles and 100 zones for the finite differencing of Eq. (8).

The basic procedure of the simulation code is to advance the marker particles one time step using Eqs. (2) and (3) with the leapfrog algorithm, ${ }^{10}$ determine the new density using Eq. (4), and then determine the new potential using Eqs. (5) and (6). Since the potential is solved on a fixed coordinate system and Eq. (4) determines the density in a Lagrangian coordinate system, we need to transform the density to that on the fixed coordinate system. This process of moving the marker particles a time step, determining the density and the potential, and taking another time step is continued until the end of the simulation. The time step is chosen so that the fastest marker particle does not move more than one fixed cell per time step. The resulting code runs satisfactorily on various desktop computers.

\section{SIMULATION RESULTS}

The code was first tested by reproducing the results of Widner et $a l^{3}$ for planar geometry and an instantaneous

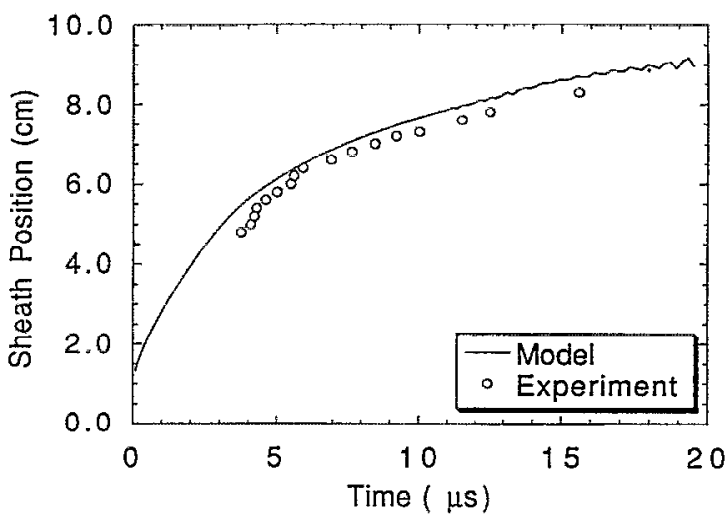

FIG. 1. Sheath radius for a cylindrical target. The plasma is argon with $n=6.8 \times 10^{9} \mathrm{~cm}^{-3}$ and $T_{e}=1.5 \mathrm{eV}$; the applied potential is $-30 \mathrm{kV}$. The experimental data is from Ref. 8 . 


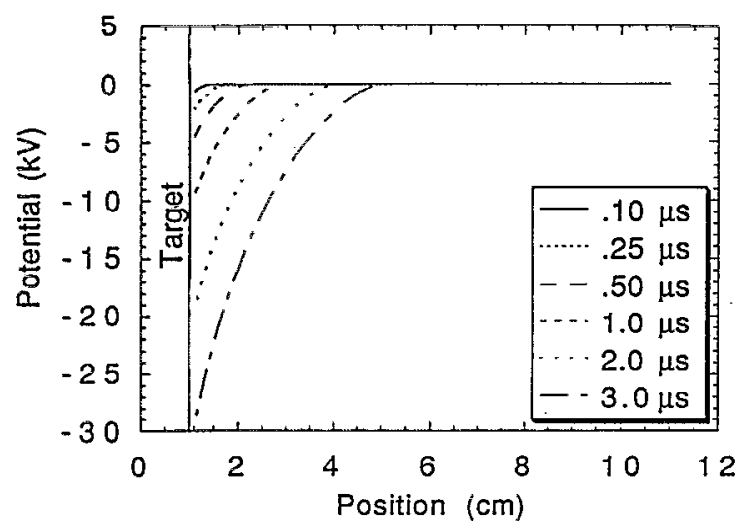

FIG. 2. Profiles of the potential during the pulse for the simulation in Fig. 1.

potential of $e \Phi / k T_{e}=-50$; the time-dependent density profile agrees very well with that given by Widner et al. In this paper we apply the code to the sheath expansion measurements of Shamim and co-workers. ${ }^{8}$ Shamim and coworkers made measurements of the location of the sheath edge using a Langmuir probe biased to measure the electron saturation current. In the simulation code, there is not a precise definition of the sheath edge. For these calculations, we estimate the sheath edge as the point where the electron density is $10 \%$ of the density far away from the target. At high applied voltage the electron density drops rapidly in the sheath so this is a reasonable measure of where the sheath starts.

We first simulate the experiment of Shamim and coworkers with a cylindrical target whose radius is $0.95 \mathrm{~cm}$; we take the outer radius $b$ to be $10.95 \mathrm{~cm}$ so that a $10-\mathrm{cm}$ annulus of plasma is being simulated. The plasma is argon with a density of $6.8 \times 10^{9} \mathrm{~cm}^{-3}$ and an electron temperature of $1.5 \mathrm{eV}$. The applied potential is zero initially and rises (in the negative sense) at a rate of $10 \mathrm{kV} / \mu$ s until it reaches the plateau value of $-30 \mathrm{kV}$. The calculated sheath position as a function of time is shown in Fig. 1 along with the experimental data of Shamim. The agreement is very good, even though the sheath radius gets to be

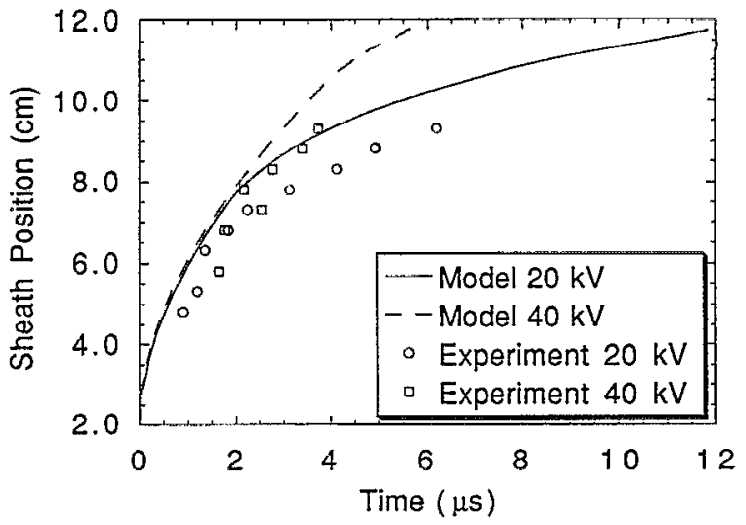

FIG. 4. Sheath radius for a spherical target. The plasma is argon with $n=5 \times 10^{8} \mathrm{~cm}^{-3}$ and $T_{e}=0.9 \mathrm{eV}$; the applied potential is -20 and $-40 \mathrm{kV}$. The experimental data is from Ref. 8 .

comparable to the half-length of the cylinder and the 1D approximation becomes questionable. Figures 2 and 3 show the evolution of the potential and ion density profiles. The rise in the ion density inside the sheath as $r$ decreases is a geometrical effect due to the convergence of the ions moving along $-r$ in cylindrical geometry. The oscillations in the density profile are numerical and decrease with more marker particles and finer mesh size. The oscillations saturate in amplitude and convect towards the target. They appear only in the density profile; the potential profile depends on a second integral of the density profile and hence the oscillations in $\Phi$ get smoothed out.

Next we simulate the spherical target experiments of Shamim and co-workers. The sphere has a $2-\mathrm{cm}$ radius; we take $a=2 \mathrm{~cm}$ and $b=12 \mathrm{~cm}$. Figure 4 shows the sheath expansion versus time and the comparison with Shamim and co-workers' data for an argon plasma with a density of $5 \times 10^{8} \mathrm{~cm}^{-3}$ and an electron temperature of $0.9 \mathrm{eV}$. Figure 5 shows a similar comparison for a density of $6 \times 10^{9}$ $\mathrm{cm}^{-3}$ and $T_{e}=1.4 \mathrm{eV}$. The agreement with the experimental data is acceptable for both the low- and high-density plasmas, although not as good as for the cylindrical simulation. The disagreement between the experimental

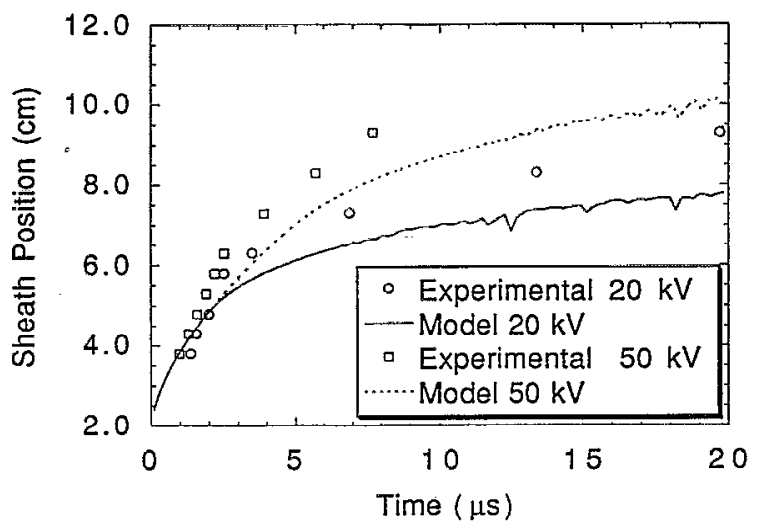

FIG. 5. Sheath radius for a spherical target. The plasma is argon with $n=6 \times 10^{9} \mathrm{~cm}^{-3}$ and $T_{\mathrm{e}}=1.4 \mathrm{eV}$; the applied potential is -20 and $-50 \mathrm{kV}$. The experimental data is from Ref. 8.
FIG. 3. Profiles of the ion density during the pulse for the simulation in Fig. 1. 


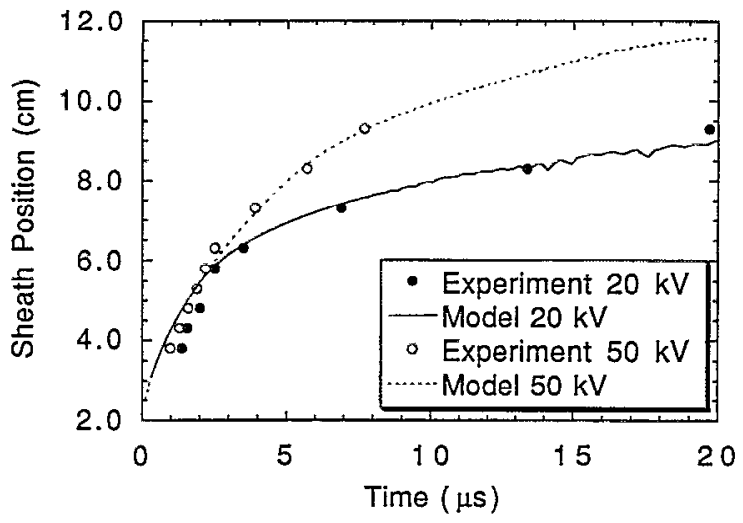

FIG. 6. Sheath radius for the same case ( $-50 \mathrm{kV}$ only) as in Fig. 5, but the density for the simulation is arbitrarily changed to $3 \times 10^{9} \mathrm{~cm}^{-3}$.

data and the simulation can be attributed to either assumptions in the simulation model which are being violated, or to errors in the experimental data. Sources of experimental errors are uncertainties in measuring the sheath position versus time and uncertainties in the reported density and temperature of the plasma. A change in the density of the plasma, which is used as input for the simulation code, will affect the calculated sheath position versus time. Figure 6 shows the comparison if the density is arbitrarily reduced from $6 \times 10^{9}$ to $3 \times 10^{9} \mathrm{~cm}^{-3}$. In this case the agreement is very good. However, the estimated uncertainty in the density is $20 \%-30 \%$, which is less than that needed to achieve agreement, so a disagreement between the measured sheath position and that given by the simulation remains. The reason for this disagreement is not understood, but the magnitude is not critical for PSII applications. The sheath expansion is rather insensitive to the electron temperature as long as the sheath velocity is faster than the ion acoustic velocity, but becomes more sensitive to the electron temperature as the steady-state sheath is approached.

The simulation code can be used to provide additional data about the plasma-source ion implantation process. The time evolution of the ion current to the target is shown in Fig. 7 for the simulation of Fig. 5 at $50 \mathrm{kV}$. The oscil-

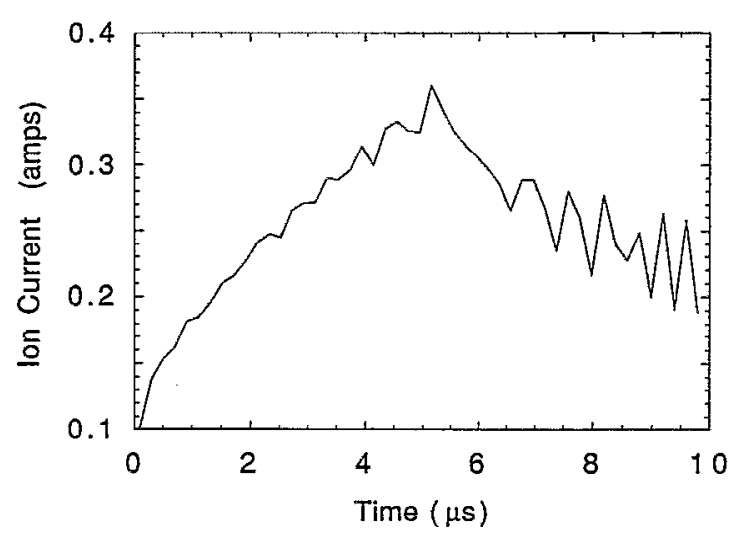

FIG. 7. Ion current to the target vs time for the simulation in Fig. 5; the applied potential is $-50 \mathrm{kV}$.

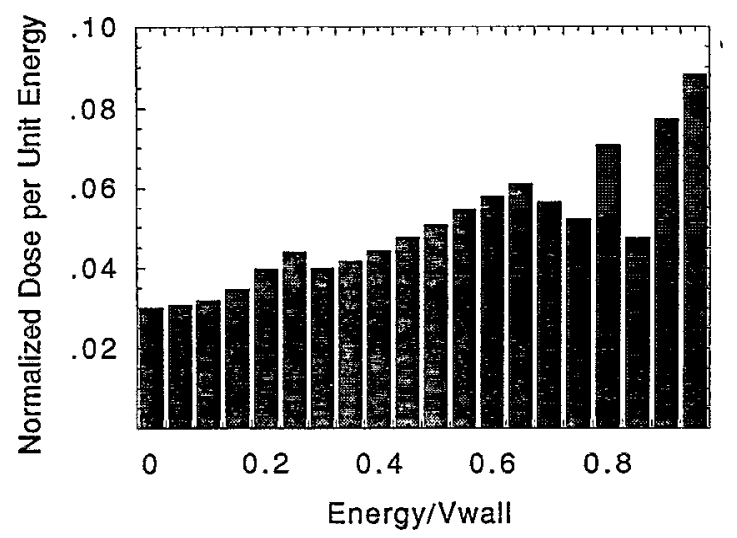

FIG. 8. Time-integrated energy spectrum of the ions hitting the surface during the first $5 \mu$ s of the pulse for the simulation in Fig. 5. The applied potential is $-50 \mathrm{kV}$ and the rise time of the pulse is $5 \mu \mathrm{s}$.

lations in the current are due to the oscillations in the density at the target. Not included in this current is the current due to secondary electron emission, which depends on the target material, surface condition, and energy of the ions hitting the target. Figures 8 and 9 show the accumulated energy spectrum (normalized to unity) of the ions incident on the surface after 5 and $10 \mu$ s, respectively, into a pulse for the same simulation. The spectrum exhibits a long, low-energy component as well as ions at the full energy corresponding to the applied potential. The low-energy component is due to ions which hit the target during the rise-time of the voltage pulse. After $10 \mu \mathrm{s}$, only about $60 \%$ of the ions have hit the target with the full energy; this fraction will increase as the pulse lengthens. Tailoring the voltage pulse allows control over the energy spectrum and may have some application in optimizing the PSII process.

Note that the simulation code is based on a first-principles calculation and does not invoke the assumption that the plasma sheath expands quasistatically at a rate given by the Child-Langmuir space-charge-limited current density, which is the assumption used in the analytical modeling by

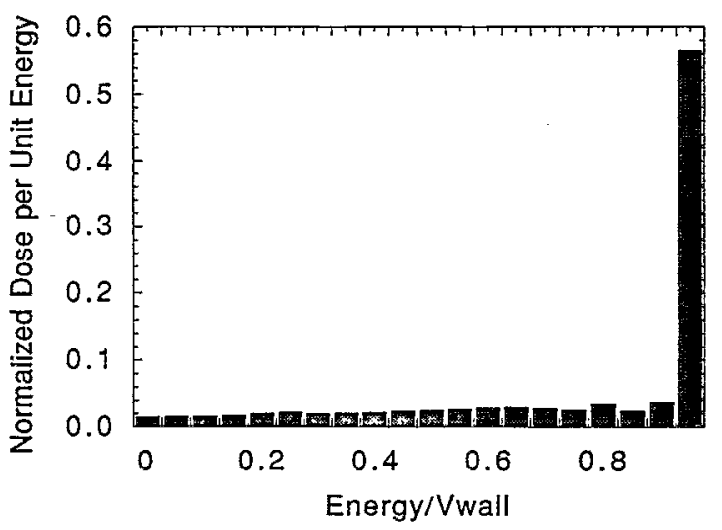

FIG. 9. Time-integrated energy spectrum of the ions hitting the surface during the first $10 \mu$ s of the pulse for the simulation in Fig. 5. The applied potential is $-50 \mathrm{kV}$ and the rise time of the pulse is $5 \mu \mathrm{s}$. 


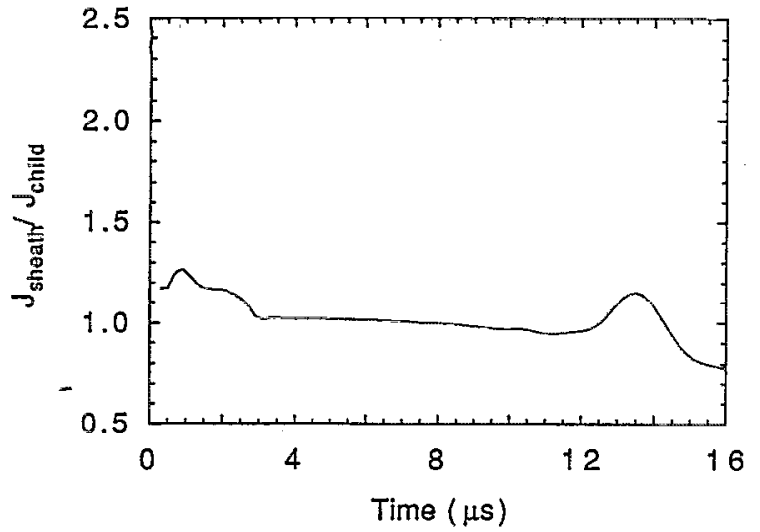

FIG. 10. Ratio of the current into the sheath to the Child-Langmuir space-charge-limited current for the simulation of Fig. 1.

Scheuer and Lieberman. Shown in Fig. 10 is the ratio of the computed current into the sheath to the current given by the Child-Langmuir law for the simulation given in Fig. 1. The ratio is close to unity, which provides support for this assumption. The differences at early and late time may be due to noise. The current into the sheath requires differentiating the sheath position versus time and hence is inherently noisy; some smoothing was done to remove the effect of noise in the sheath position.

In these simulations, the sheath expansion rate is large and the plasma has not yet formed a presheath. For longer pulse times or higher-density plasmas, the sheath can essentially stop and a density rarefaction wave propagates ahead of the sheath, forming the presheath. An example of this is shown in Figs. 11 and 12. This simulation is for a 2.5 -cm-diam spherical target immersed in a nitrogen plasma with a density of $2 \times 10^{10} \mathrm{~cm}^{-3}$, an electron temperature of $5 \mathrm{eV}$, and an applied potential of $-20 \mathrm{kV}$. The sheath effectively stops after about $5 \mu \mathrm{s}$ and the presheath starts forming. Figure 12 shows the resulting electron and ion density profiles at $14.8 \mu \mathrm{s}$. The plasma is quasineutral for radii greater than about $4 \mathrm{~cm}$ but the plasma density is

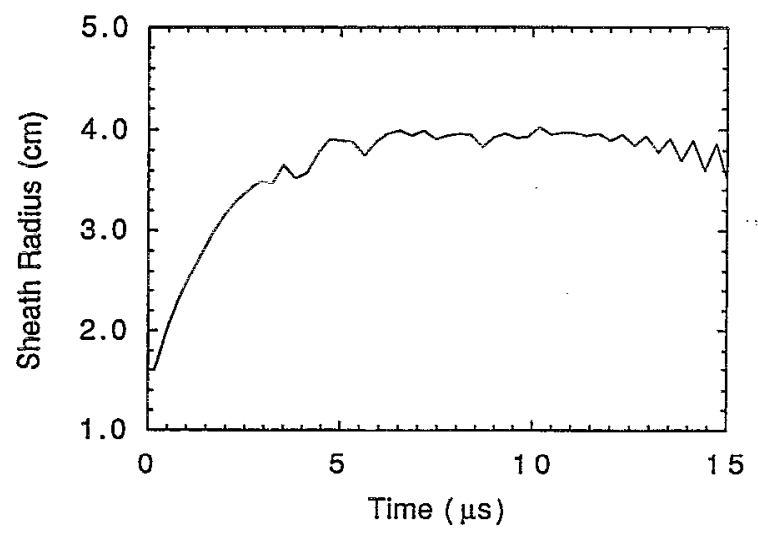

FIG. 11. Sheath radius for a $2.5-\mathrm{cm}$-diam spherical target. The plasma is nitrogen with $n=2 \times 10^{10} \mathrm{~cm}^{-3}$ and $T_{e}=5 \mathrm{eV}^{-3}$ the applied potential is $-20 \mathrm{kV}$

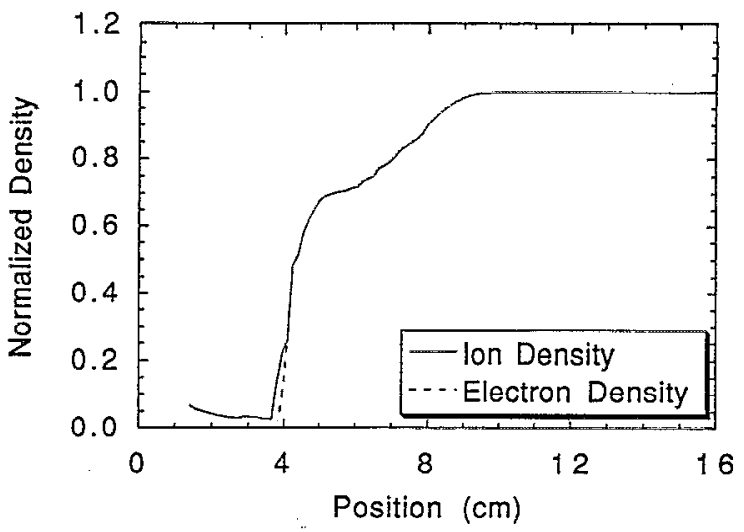

FIG. 12. Electron and ion density profile at $t=14.8 \mu$ s for the simulation of Fig. 11.

still varying significantly for radii up to about $10 \mathrm{~cm}$. This stopping of the sheath has been seen in experiments by Hutchings, Collins, and Tendys ${ }^{12}$ for plasma parameters near to those used in the simulation.

\section{SUMMARY}

We have developed a one-dimensional time-dependent code which simulates the sheath evolution during a voltage pulse in plasma-source ion implantation. The ions are treated as cold and collisionless; the electrons are assumed to satisfy the Boltzmann approximation. The self-consistent potential is calculated from Poisson's equation. The agreement between the simulation and the experimental results of Shamim is good. The simulation model is being extended to two-dimensions to determine the effect of the geometry on the uniformity of the implanted ion dose.

\section{ACKNOWLEDGMENTS}

The authors gratefully acknowledge helpful discussions with J. T. Scheuer, M. Shamim, and J. R. Conrad. This research was supported by the Wisconsin Alumni Research Foundation and the National Science Foundation.

' J. R. Conrad, J. L. Radke, R. A. Dodd, F. J. Worzala, and N. C. Tran, J. Appl. Phys. 62, 4591 (1987).

${ }^{2}$ J. R. Conrad, R. A. Dodd, F. J. Worzala, and X. Qui, Surf. Coatings Technol. 36, 927 (1988).

${ }^{3}$ M. Widner, I. Alexeff, W. D. Jones, and K. E. Lonngren, Phys. Fluids 13, 2532 (1970).

${ }^{4}$ J. K. Chester, J. Vac. Sci. Technol. 37, 2 (1970).

${ }^{5}$ M. A. Lieberman, J. Appl. Phys. 66, 2927 (1989).

${ }^{6} J$. T. Scheuer, M. Shamim, and J. R. Conrad, J. Appl. Phys. 67, 1241 (1990).

${ }^{7}$ R. A. Stewart and M. Lieberman, J. Appl. Phys. 70, 3481 (1991).

${ }^{8}$ M. Shamim, J. T. Scheuer, and J. R. Conrad, J. Appl. Phys. 69, 2904 (1991).

${ }^{9}$ R. W. Hockney and J. W. Eastwood, Computer Simulation Using Particles (Adam Hilger, New York, 1988), p. 185.

${ }^{10} \mathrm{C}$. K. Birdsall and A. B. Langdon, Plasma Physics vîa Computer Simulation (McGraw-Hill, New York, 1985), p. 12.

${ }^{11} M$. Shamim and J. R. Conrad (private communication).

${ }^{12}$ R. Hutchings, G. A. Collins, and J. Tendys, in Seventh International Conference on Surface Modification of Mctals by Ion Beams, Washington, DC July 15-19, 1991. 